\title{
Inflammatory Bowel Disease and Thrombosis: A National Inpatient Sample Study
}

\author{
Jessica B. Cohen ${ }^{1,2}$ Diane M. Comer ${ }^{3}$ Jonathan G. Yabes ${ }^{3}$ Margaret V. Ragni,2 \\ ${ }^{1}$ Department of Medicine, Division of Hematology/Oncology, \\ University of Pittsburgh Medical Center and Hemophilia Center of \\ Western Pennsylvania, Pittsburgh, Pennsylvania, United States \\ 2 University of Pittsburgh, Pittsburgh, Pennsylvania, United States \\ 3 University of Pittsburgh Center for Research on Health Care Data

\begin{abstract}
Address for correspondence Margaret V. Ragni, MD, MPH, Division of Hematology Oncology, Department of Medicine and Clinical Translational Science, Hemophilia Center of Western Pennsylvania, University of Pittsburgh, 3636 Boulevard of the Allies, Pittsburgh,
\end{abstract} \\ PA 15213-4306, United States (e-mail: ragni@pitt.edu).
} Center, Pittsburgh, Pennsylvania, United States

TH Open 2020;4:e51-e58.

\begin{abstract}
Keywords

- inflammatory bowel disease

- malignancy

- malnutrition

- steroids

- thrombosis

Introduction Thrombosis is more common in inflammatory bowel disease (IBD) patients than the general population, but disease-specific correlates of thrombosis remain unclear. Methods We performed a retrospective analysis of discharge data from the National Inpatient Sample between 2009 and 2014, using International Disease Classification codes to identify IBD and non-IBD patients with or without thrombosis. We used NIS-provided discharge-level weights to reflect prevalence estimates. Categoric variables were analyzed by Rao-Scott Chi-square test, continuous variables by weighted simple linear regression, and covariates associated with thrombosis by weighted multivariable logistic regression. Results Thrombosis prevalence in IBD was significantly greater than in non-IBD, 7.52 versus $4.54 \%, p<0.0001$. IBD patients with thrombosis were older and more likely to be Caucasian than IBD without thrombosis, each $p<0.001$. Thrombosis occurred most commonly in the mesenteric vein. Thrombotic risk factors in IBD include surgery, ports, malignancy, dehydration, malnutrition, and steroids at 53.7, 13.2, 13.1, 12.4, 8.9, and $8.2 \%$, respectively. Those with thrombosis had greater severity of illness, 1.42 versus 0.96 ; length of stay, 7.7 versus 5.5 days; and mortality, 3.8 versus $1.5 \%$; all $p<0.0001$. Adjusting for age and comorbidity, odds ratios for predictors of thrombosis included ports, steroids, malnutrition, and malignancy at $1.73,1.61,1.34$, and 1.13 , respectively, while Asian race, 0.61 , was protective, each $p<0.001$.

Conclusion Thrombosis prevalence is 1.7 -fold greater in IBD than non-IBD patients. Adjusting for age and comorbidity, the odds ratio for thrombosis in IBD was $73 \%$ higher with ports, $61 \%$ higher with steroids, $34 \%$ with malnutrition, and $13 \%$ with malignancy. Whether long-term anticoagulation would benefit the latter is unknown.
\end{abstract}

\section{Introduction}

Inflammatory bowel disease is a chronic inflammatory disorder of the gastrointestinal (GI) mucosa including Crohn's disease (CD) and ulcerative colitis (UC). An estimated 1.4 million people in the United States currently suffer from IBD, and the incidence is increasing with time. ${ }^{1-3}$ These disorders are characterized by defective immune regulation in which T-cells in the GI mucosa secrete inflammatory cytokines. ${ }^{4,5}$ The subsequent inflammatory response, both acute and chronic, induces a prothrombotic state which is mediated by an increase in procoagulant factors and a decrease in natural anticoagulants and in fibrinolytic activity. ${ }^{6}$ More specifically, there is an upregulation of tissue factor and an received

December 20, 2019

accepted

April 7, 2020
DOI https://doi.org/

10.1055/s-0040-1710506. ISSN 2512-9465. (c) 2020 Georg Thieme Verlag KG
Stuttgart · New York

License terms

(c) (1) 
increase in the platelet count that promotes thrombosis; at the same time, decreased expression of tissue plasminogen activator and increased levels of plasminogen activator inhibitor-1 contribute to decreased fibrinolysis. ${ }^{7,8}$ Under these conditions, development of venous thromboembolism (VTE) is one of the most common extrasystemic manifestations of IBD, with a risk 2 to 3 times greater than in the general population. Moderate and severe disease flares, hospitalization, and surgery appear to increase thrombosis risk further. ${ }^{9-11}$

Current guidelines suggest the use of pharmacologic VTE prophylaxis in IBD patients admitted to the hospital for any reason, especially if the admission is for a disease flare, even if accompanied by nonsevere GI bleeding, as the latter is not a contraindication to anticoagulation. ${ }^{12}$ However, surveys of both American and Canadian gastroenterologists reveal a lack of institutional standards, with a wide variation in the application of these guidelines, many of which are based on low-quality evidence. ${ }^{13,14}$ Morbidity and mortality remain elevated for these patients, and it is unclear what additional risk factors may place patients at risk for thrombosis. We analyzed the National Inpatient Sample to further characterize the prevalence and risk factors for thrombosis in hospitalized patients with IBD.

\section{Methods}

\section{Study Design and Data Source}

This was a retrospective analysis of discharge data from the National Inpatient Sample (NIS) between January 1, 2009 and December 31, 2014. The NIS is an all-payer database that approximates a $20 \%$ stratified sample of discharges from U.S. community hospitals participating in the Healthcare Cost and Utilization Project. ${ }^{15}$ It contains unweighted data from more than 7 million yearly hospital stays, and once weighted, estimates approximately 35 million stays. It includes deidentified clinical and nonclinical elements such as primary and secondary diagnoses, patient demographics, payment source, length of stay, and severity and comorbidity measures. Data during this time period were classified by the International Classification of Diseases, Ninth Revision (ICD9) diagnostic codes.

\section{Populations}

Patient discharges, both with and without IBD and with and without thrombosis, were identified using ICD-9 diagnostic codes 555.0 (regional enteritis) and 556.0 (ulcerative colitis). Codes used to identify venous thrombosis included deep venous thrombosis $(451.11,451.19,451.2,451.81,451.83$, $451.9,453.40,453.41,453.42,453.8$, and V12.51), pulmonary embolism $(415.11,415.13$, and 415.19$)$, portal vein thrombosis (452.0), splenic vein thrombosis (444.89), mesenteric vein thrombosis (557.0 and 557.1), hepatic vein thrombosis (453.0), and intracranial venous sinus thrombosis (325.0). Arterial thrombosis was identified using the following codes: arterial thrombosis/embolism (444), cerebral artery embolism (434.1), cerebral artery thrombosis (434), and retinal artery occlusion (362.3).

\section{Potential Risk Factors}

The frequency of potential risk factors for thrombosis in patients with IBD was evaluated and identified using diagnosis codes: dehydration $(276.5,639.5$, and 998.0), malnutrition $(263.0,263.1,263.8$, and 263.9), ports/central venous catheters (996.1 and 38.93), long-term steroid use (V58.65), postoperative state (V45, 998.9), malignancy (140-239), pregnancy (650), hormone therapy (V25.01, V25.09, and V25.8), immobilization (V49.89), trauma (800-959), and surgical procedures (general procedures: 01-86; abdominal: 45 , 46, and 48; and orthopaedic: $76-84$ ).

\section{Comorbidities}

General comorbidities were evaluated using the following codes: diabetes (250-250.3 and 250.7), obesity (278.0), smoking (305.1 and V15.82), hypertension (401-405), hyperlipidemia (272.0, 272.2, and 272.4), cardiac disease (410-414), renal failure $(403,404,585$, and 586$)$, liver disease (571.8, 571.9, and 572.8), cirrhosis (571.5), hepatitis C (070.41, 070.44, 070.51, 070.54, 070.70, and 070.71), $\operatorname{HIV}(042,079.53,795.71$, and V08), and transfusion (blood or blood products: V582, 5187, 9647, 9996, 9997, and E8760; platelets: 99.05; and coagulation factors: 99.06). Deyo's modification of the Charlson's comorbidity index was used to assess the severity of illness between groups; by assigning weights to 16 diseases based on the strength of their association with mortality, the index controls for confounding.

\section{Statistical Analysis}

Thrombosis prevalence was estimated among admissions with and without IBD. The two groups were compared with respect to patient characteristics (age, race, and gender), insurance type, length of stay, inpatient mortality, and risk factors for thrombosis. These variables were further compared with and without thrombosis within each IBD group. Univariate analyses for between-group comparisons used Rao-Scott Chi-square test for categorical variables (e.g., gender and risk factors) and weighted simple linear regression for continuous variables (e.g., age). Weighted multivariable logistic regression was performed to identify factors associated with thrombosis among those with IBD, controlling for age and comorbidity. Odds ratios (ORs) and 95\% confidence intervals (CIs) were calculated. Multivariable logistic regression was performed to identify factors associated with thrombosis among those with IBD. The multivariable model included covariates selected based on clinical and statistical significance. All analyses used discharge-level weights provided by NIS to reflect national estimates. All analyses were conducted in SAS (SAS version 9.4, SAS corporation), and $p$ values $<0.05$ were considered statistically significant.

\section{Results}

\section{Admission Characteristics}

During the 6-year period from January 1, 2009 to December 31,2014 , an unweighted total of $45,123,086$ admissions was collected of which 374,315 included a diagnosis code for IBD. The prevalence of thrombosis in patients with IBD 
(7.51\%, $n=28,155)$ was 1.7 times higher than the prevalence of thrombosis in patients without $\operatorname{IBD}(4.53 \%, n=2,029,915)$, $p<0.0001$. IBD patients had an increased prevalence of thrombotic risk factors including general surgery, abdominal surgery, dehydration, ports/central venous catheters, mal- nutrition, and long-term steroid use, all $p<0.0001$, as compared with non-IBD patients ( - Table 1 ). Markers of overall health, including the Charlson's score (9.99 vs. 1.25, $p<0.0001)$ and length of stay (5.63 vs. 4.58 days) were significantly worse, while inpatient mortality (1.66 vs.

Table 1 Characteristics of IBD and non-IBD admissions from 2009 to 2014

\begin{tabular}{|c|c|c|c|}
\hline \multirow[t]{2}{*}{ Baseline characteristics } & IBD & No IBD & \multirow[t]{2}{*}{$p$-Value } \\
\hline & \multicolumn{2}{|c|}{ Percent of mean (SE) } & \\
\hline \multicolumn{4}{|l|}{ No. of hospital admissions } \\
\hline Actual & 374,315 & $44,748,771$ & \\
\hline Weighted & $1,860,564$ & $222,614,880$ & \\
\hline \multicolumn{4}{|l|}{ Patient characteristics } \\
\hline Age (y) & $50.97(0.03)$ & $48.66(0.00)$ & $<0.0001$ \\
\hline \multicolumn{3}{|l|}{ Race } & \multirow[t]{5}{*}{$<0.0001$} \\
\hline Caucasian & 79.82 & 65.76 & \\
\hline African American & 10.67 & 14.98 & \\
\hline Asian & 1.03 & 2.69 & \\
\hline Other & 8.48 & 16.57 & \\
\hline \multicolumn{3}{|l|}{ Gender } & \multirow[t]{3}{*}{$<0.0001$} \\
\hline Female & 56.86 & 57.72 & \\
\hline Male & 43.14 & 42.28 & \\
\hline \multicolumn{4}{|l|}{ Thrombosis } \\
\hline Any thrombosis & 7.51 & 4.53 & $<0.0001$ \\
\hline Mesenteric vein thrombosis & 0.56 & 0.18 & $<0.0001$ \\
\hline Pulmonary embolism & 0.49 & 0.48 & 0.4276 \\
\hline Deep venous thrombosis & 0.47 & 0.36 & $<0.0001$ \\
\hline Portal vein thrombosis & 0.19 & 0.06 & $<0.0001$ \\
\hline Cerebral artery embolism & 0.16 & 0.25 & $<0.0001$ \\
\hline Other/splenic vein thrombosis & 0.04 & 0.02 & $<0.0001$ \\
\hline Cerebral artery thrombosis & 0.03 & 0.04 & 0.0144 \\
\hline Intracranial venous sinus thrombosis & 0.03 & 0.01 & $<0.0001$ \\
\hline Retinal occlusion (artery or vein) & 0.03 & 0.04 & 0.0011 \\
\hline Hepatic vein thrombosis (Budd-Chiari syndrome) & 0.02 & 0.01 & $<0.0001$ \\
\hline \multicolumn{4}{|l|}{ Risk factors } \\
\hline General surgical procedures & 52.01 & 50.23 & $<0.0001$ \\
\hline Abdominal surgery & 26.21 & 4.89 & $<0.0001$ \\
\hline Dehydration & 13.15 & 6.31 & $<0.0001$ \\
\hline Malignancy & 9.48 & 10.28 & $<0.0001$ \\
\hline Ports/central venous catheters & 8.07 & 4.10 & $<0.0001$ \\
\hline Malnutrition & 6.03 & 2.46 & $<0.0001$ \\
\hline Long-term steroid use & 5.71 & 0.99 & $<0.0001$ \\
\hline Orthopedic surgery & 4.94 & 8.33 & $<0.0001$ \\
\hline Trauma & 4.15 & 6.95 & $<0.0001$ \\
\hline Charlson's score comorbidity index & $9.99(0.00)$ & $1.25(0.00)$ & $<0.0001$ \\
\hline Length of stay & $5.63(0.01)$ & $4.58(0.00)$ & $<0.0001$ \\
\hline Discharge status (inpatient mortality) & 1.66 & 1.90 & $<0.0001$ \\
\hline
\end{tabular}

Abbreviations: IBD, inflammatory bowel disease; SE, standard error. 
$1.90 \%, p<0.0001)$ was significantly lower in IBD patients compared with patients without IBD ( - Table $\mathbf{1}$ ).

Among patients with IBD, those with thrombosis were significantly older ( 57.48 vs. 50.44 years, $p<0.0001$ ), more likely to be Caucasian ( 81.54 vs. $79.68 \%, p<0.0001$ ), and less likely to be Asian ( 0.62 vs. $1.06 \%, p<0.0001$ ), than those without thrombosis. There was no significant gender effect on thrombosis prevalence in IBD patients (-Table 2 ).
Among venous thromboses, mesenteric vein thrombosis was the most common type. Patients with IBD were nearly twice as likely to have mesenteric vein thrombosis ( 7.45 vs. $3.94 \%, p<0.0001$ ) or portal vein thrombosis ( 2.53 vs. $1.42 \%$, $p<0.0001)$, as patients without IBD. By contrast, pulmonary embolism (6.46 vs. 10.50\%) and deep venous thrombosis (6.21 vs. 7.96\%) were significantly lower in IBD than in those without IBD, each $p<0.0001$. Among arterial thromboses,

Table 2 Patient characteristics and risk factors for thrombosis by IBD and thrombosis status

\begin{tabular}{|c|c|c|c|c|c|c|}
\hline & \multicolumn{2}{|c|}{ IBD } & \multicolumn{2}{|c|}{ No IBD } & \multirow[b]{2}{*}{1 vs. 2} & \multirow[b]{2}{*}{1 vs. 3} \\
\hline & Thrombosis & $\begin{array}{l}\text { No } \\
\text { thrombosis }\end{array}$ & Thrombosis & $\begin{array}{l}\text { No } \\
\text { thrombosis }\end{array}$ & & \\
\hline & Group 1 & Group 2 & Group 3 & Group 4 & \multirow{2}{*}{\multicolumn{2}{|c|}{$p$-Value }} \\
\hline & \multicolumn{4}{|c|}{ Percent or mean (SE) } & & \\
\hline \multicolumn{7}{|l|}{ No. of hospital admissions } \\
\hline Actual & 28,155 & 346,160 & $2,029,915$ & $42,718,856$ & & \\
\hline Weighted & 139,767 & $1,720,797$ & $10,092,645$ & $212,522,234$ & & \\
\hline Age $(y)$ & $57.48(0.11)$ & $50.44(0.03)$ & $65.01(0.01)$ & $47.88(0.00)$ & $<0.0001$ & $<0.0001$ \\
\hline$<18$ & 0.75 & 3.80 & 0.65 & 16.50 & & \\
\hline $18-40$ & 18.83 & 30.99 & 8.93 & 21.93 & & \\
\hline$>40$ & 80.42 & 65.22 & 90.42 & 61.56 & & \\
\hline \multicolumn{5}{|l|}{ Race } & \multirow[t]{5}{*}{$<0.0001$} & \multirow[t]{5}{*}{$<0.0001$} \\
\hline Caucasian & 81.54 & 79.68 & 73.2 & 65.4 & & \\
\hline African American & 10.98 & 10.65 & 16.53 & 14.90 & & \\
\hline Asian & 0.62 & 1.06 & 1.20 & 2.77 & & \\
\hline Other & 6.86 & 8.61 & 9.06 & 16.93 & & \\
\hline \multicolumn{5}{|l|}{ Gender } & \multirow[t]{3}{*}{0.0816} & \multirow[t]{3}{*}{0.0002} \\
\hline Female & 56.37 & 56.90 & 55.25 & 57.84 & & \\
\hline Male & 43.63 & 43.10 & 44.75 & 42.16 & & \\
\hline \multicolumn{7}{|l|}{ Type IBD } \\
\hline Regional enteritis & 60.15 & 63.92 & 0 & 0 & $<0.0001$ & \\
\hline Ulcerative colitis & 40.14 & 36.45 & 0 & 0 & $<0.0001$ & \\
\hline \multicolumn{7}{|l|}{ Thrombosis (\%) } \\
\hline Pulmonary embolism & 6.46 & 0 & 10.50 & 0 & & $<0.0001$ \\
\hline Deep venous thrombosis & 6.21 & 0 & 7.96 & 0 & & $<0.0001$ \\
\hline Arterial thrombosis/arterial embolism & 0.03 & 0 & 0.09 & 0 & & 0.0016 \\
\hline \multicolumn{7}{|l|}{ Intraabdominal thrombosis (\%) } \\
\hline Mesenteric vein thrombosis & 7.45 & 0 & 3.94 & 0 & & $<0.0001$ \\
\hline Portal vein thrombosis & 2.53 & 0 & 1.42 & 0 & & $<0.0001$ \\
\hline Other/splenic vein thrombosis & 0.54 & 0 & 0.51 & 0 & & 0.3792 \\
\hline $\begin{array}{l}\text { Hepatic vein thrombosis } \\
\text { (Budd-Chiari syndrome) }\end{array}$ & 0.30 & 0 & 0.15 & 0 & & $<0.0001$ \\
\hline \multicolumn{7}{|l|}{ Other thromboembolism } \\
\hline Cerebral artery embolism & 2.16 & 0 & 5.48 & 0 & & $<0.0001$ \\
\hline Cerebral artery thrombosis & 0.42 & 0 & 0.87 & 0 & & $<0.0001$ \\
\hline Retinal occlusion (artery or vein) & 0.36 & 0 & 0.82 & 0 & & $<0.0001$ \\
\hline Intracranial venous sinus thrombosis & 0.35 & 0 & 0.26 & 0 & & 0.0021 \\
\hline
\end{tabular}


Table 2 (Continued)

\begin{tabular}{|c|c|c|c|c|c|c|}
\hline & \multicolumn{2}{|c|}{ IBD } & \multicolumn{2}{|c|}{ No IBD } & \multirow[b]{2}{*}{1 vs. 2} & \multirow[b]{2}{*}{1 vs. 3} \\
\hline & Thrombosis & $\begin{array}{l}\text { No } \\
\text { thrombosis }\end{array}$ & Thrombosis & $\begin{array}{l}\text { No } \\
\text { thrombosis }\end{array}$ & & \\
\hline \multicolumn{7}{|l|}{ Risk factors/comorbidities } \\
\hline General surgery & 53.73 & 51.87 & 45.61 & 50.45 & $<0.0001$ & $<0.0001$ \\
\hline Abdominal surgery & 24.72 & 26.33 & 8.16 & 4.73 & $<0.0001$ & $<0.0001$ \\
\hline Ports/central venous catheters & 13.19 & 7.65 & 8.72 & 3.88 & $<0.0001$ & $<0.0001$ \\
\hline Malignancy & 13.10 & 9.18 & 18.62 & 9.88 & $<0.0001$ & $<0.0001$ \\
\hline Dehydration & 12.43 & 13.21 & 7.75 & 6.24 & 0.0002 & $<0.0001$ \\
\hline Malnutrition & 8.87 & 5.79 & 4.92 & 2.34 & $<0.0001$ & $<0.0001$ \\
\hline Long-term steroid use & 8.20 & 5.51 & 2.31 & 0.93 & $<0.0001$ & $<0.0001$ \\
\hline Orthopedic surgery & 5.19 & 4.92 & 7.51 & 8.36 & 0.0403 & $<0.0001$ \\
\hline Trauma & 5.03 & 4.08 & 7.42 & 6.93 & $<0.0001$ & $<0.0001$ \\
\hline \multicolumn{7}{|l|}{ Medical conditions } \\
\hline Hypertension & 45.17 & 36.61 & 62.51 & 41.79 & $<0.0001$ & $<0.0001$ \\
\hline Smoking & 26.65 & 26.09 & 26.43 & 19.93 & 0.0427 & 0.4201 \\
\hline Hyperlipidemia & 21.50 & 17.77 & 33.59 & 22.62 & $<0.0001$ & $<0.0001$ \\
\hline Cardiac disease & 16.61 & 12.73 & 26.24 & 17.76 & $<0.0001$ & $<0.0001$ \\
\hline Diabetes & 14.95 & 12.14 & 22.82 & 16.32 & $<0.0001$ & $<0.0001$ \\
\hline Renal failure & 13.55 & 8.90 & 18.05 & 10.83 & $<0.0001$ & $<0.0001$ \\
\hline Obesity & 10.44 & 7.55 & 14.85 & 9.34 & $<0.0001$ & $<0.0001$ \\
\hline Cirrhosis & 1.72 & 1.25 & 1.18 & 0.79 & $<0.0001$ & $<0.0001$ \\
\hline Liver disease & 1.66 & 1.47 & 1.07 & 0.76 & 0.0118 & $<0.0001$ \\
\hline Hepatitis C & 1.32 & 1.49 & 1.69 & 1.61 & 0.0187 & $<0.0001$ \\
\hline HIV & 0.48 & 0.48 & 0.74 & 0.62 & 0.9626 & $<0.0001$ \\
\hline \multicolumn{7}{|l|}{ Transfusion } \\
\hline Blood or blood products & 0.01 & 0.01 & 0.02 & 0.01 & 0.6889 & 0.4667 \\
\hline Platelets & 1.28 & 0.70 & 1.20 & 0.67 & $<0.0001$ & 0.2550 \\
\hline Coagulation factors & 0.05 & 0.02 & 0.05 & 0.03 & 0.0153 & 0.7531 \\
\hline Charlson's comorbidity index & $1.42(0.01)$ & $0.96(0.00)$ & $2.17(0.00)$ & $1.20(0.00)$ & $<0.0001$ & $<0.0001$ \\
\hline Length of Stay & $7.75(0.06)$ & $5.46(0.01)$ & $6.72(0.01)$ & $4.48(0.00)$ & $<0.0001$ & $<0.0001$ \\
\hline Discharge Status (inpatient mortality) & 3.76 & 1.49 & 4.47 & 1.78 & $<0.0001$ & $<0.0001$ \\
\hline
\end{tabular}

Abbreviations: IBD, inflammatory bowel disease; SE, standard error.

cerebral arterial embolism (2.16 vs. $5.48 \%, p<0.0001)$ and cerebral arterial thrombosis ( 0.42 vs. $0.96 \%, p<0.0001)$ were also lower in IBD patients (-Table 2).

\section{Univariate Analysis}

Among patients with IBD, those with thrombosis were older $(p<0.0001)$ and more likely to be Caucasian $(p<0.0001)$ than those without thrombosis (-Table 2). Patients who developed thrombosis were more likely to have had a surgical procedure (53.73 vs. $51.87 \%$ ), a port or central venous catheter (CVC; 13.19 vs. $7.65 \%$ ), malignancy (13.10 vs. $9.18 \%$ ), malnutrition ( 8.87 vs. $5.79 \%$ ), received long-term steroids ( 8.20 vs. $5.51 \%$ ), or suffered trauma (5.03 vs. $4.08 \%$ ), all $p<0.0001$, compared with patients without thrombosis. Patients with thrombosis were slightly more likely to have had orthopaedic surgical procedures (5.19 vs. $4.92 \%)$, $p=0.04$, but less likely to be dehydrated (12.43 vs. $13.21 \%$ ) or to have had abdominal surgery (24.72 vs. 26.33\%), $p<0.0001$ (-Table 2).

Among all thrombosis patients, comorbid conditions including cirrhosis and liver disease were significantly more common in IBD patients, while hypertension, hyperlipidemia, cardiac disease, diabetes, renal failure, obesity, hepatitis C, and HIV were significantly less common, as compared with non-IBD patients; all $p<0.0001$. Smoking was not different between the groups, while markers of overall health, including the Charlson's score (1.42 vs. $2.17, p<0.0001)$ and inpatient mortality (3.76 vs. $4.47 \%, p<0.0001$ ) were significantly less severe and length of stay ( 7.75 vs. 6.72 days, $p<0.0001$ ) longer in IBD patients with thrombosis (-Table 2 ). 
Table 3 Multivariable logistic regression odds ratios for thrombosis in IBD

\begin{tabular}{|l|l|l|}
\hline Covariate & OR $(95 \% \mathrm{Cl})$ & $p$-Value \\
\hline Port & $1.73(1.66-1.80)$ & $<0.0001$ \\
\hline Long-term steroid use & $1.61(1.54-1.69)$ & $<0.0001$ \\
\hline Malnutrition & $1.34(1.27-1.40)$ & $<0.0001$ \\
\hline Race Caucasian & Reference & \\
\hline African American & $1.14(1.10-1.19)$ & $<0.0001$ \\
\hline Asian & $0.61(0.52-0.72)$ & $<0.0001$ \\
\hline Other & $0.88(0.84-0.93)$ & $<0.0001$ \\
\hline Malignancy & $1.13(1.08-1.18)$ & $<0.0001$ \\
\hline $\begin{array}{l}\text { Charlson's } \\
\text { comorbidity score }\end{array}$ & $1.09(1.08-1.10)$ & $<0.0001$ \\
\hline Age & $1.02(1.01-1.02)$ & $<0.0001$ \\
\hline Trauma & $1.02(0.96-1.08)$ & 0.4993 \\
\hline Hypertension & $1.01(0.98-1.05)$ & 0.3560 \\
\hline General surgery & $0.96(0.93-0.98)$ & 0.0021 \\
\hline Renal failure & $0.95(0.90-0.99)$ & 0.0285 \\
\hline Hyperlipidemia & $0.94(0.90-0.97)$ & 0.0002 \\
\hline Dehydration & $0.86(0.83-0.90)$ & $<0.0001$ \\
\hline
\end{tabular}

Abbreviations: $\mathrm{Cl}$, confidence interval; IBD, inflammatory bowel disease; OR, odds ratio.

\section{Multivariable Logistic Regression}

After adjusting for age and severity of illness with multivariable logistic regression, risk factors remaining significant for thrombosis in IBD patients included the presence of a port/ central venous catheter $(\mathrm{OR}=1.73,95 \% \mathrm{Cl}$ : 1.66-1.80), longterm steroid use $(\mathrm{OR}=1.61,95 \% \mathrm{CI}: 1.54-1.69)$, malnutrition $(\mathrm{OR}=1.34,95 \% \mathrm{CI}: 1.27-1.40)$, and malignancy $(\mathrm{OR}=1.13$, 95\% CI: 1.08-1.18; - Table 3). By contrast, Asian race $(\mathrm{OR}=0.61,95 \% \mathrm{CI}: 0.52-0.72)$ appeared to be protective against thrombosis. Several factors, while statistically significant, did not appear to be clinically strong risk or protective factors, including general surgery, renal failure, hyperlipidemia, and dehydration (-Table 3). Further multivariate analysis, after adjusting for potential confounders, that is, the variables in - Table 3, confirmed the difference in thrombosis rate between IBD and non-IBD patients (-Table 4), similar to the model in which only age was adjusted.

\section{Discussion}

This cross-sectional study of discharges in the NIS cohort demonstrates that the prevalence of thrombosis is 1.7-fold greater in those with IBD as compared with those without IBD, consistent with previous studies. ${ }^{1-3,9-11}$ The most common site was intra-abdominal, that is, mesenteric vein thrombosis, as previously reported. ${ }^{16}$ IBD patients with thrombosis were younger, and, accordingly also had lower comorbidity score and lower in-hospital mortality. Thrombosis-specific risk factors, including steroid use, malnutrition, dehydration, port use, abdominal surgery, and general surgery, were all significantly
Table 4 Multivariable logistic regression odds ratios for thrombosis in all patients

\begin{tabular}{|l|l|l|}
\hline Covariate & OR $(95 \% \mathrm{CI})$ & $p$-Value \\
\hline IBD $^{\text {a }}$ & $1.74(1.71-1.76)$ & $<0.0001$ \\
\hline Port & $2.05(2.03-2.06)$ & $<0.0001$ \\
\hline Long-term steroid use & $1.74(1.72-1.76)$ & $<0.0001$ \\
\hline Malnutrition & $1.28(1.27-1.29)$ & $<0.0001$ \\
\hline Race Caucasian & Reference & \\
\hline African American & $1.19(1.18-1.19)$ & $<0.0001$ \\
\hline Asian & $0.50(0.50-0.51)$ & $<0.0001$ \\
\hline Other & $0.70(0.70-0.71)$ & $<0.0001$ \\
\hline Malignancy & $1.30(1.29-1.31)$ & $<0.0001$ \\
\hline Hypertension & $1.12(1.12-1.13)$ & $<0.0001$ \\
\hline $\begin{array}{l}\text { Charlson's } \\
\text { comorbidity score }\end{array}$ & $1.12(1.11-1.12)$ & $<0.0001$ \\
\hline Age & $1.02(1.02-1.02)$ & $<0.0001$ \\
\hline Hyperlipidemia & $1.01(1.01-1.02)$ & $<0.0001$ \\
\hline Trauma & $0.92(0.91-0.92)$ & $<0.0001$ \\
\hline Dehydration & $0.86(0.85-0.86)$ & $<0.0001$ \\
\hline General surgery & $0.80(0.80-0.81)$ & $<0.0001$ \\
\hline Renal failure & $0.79(0.79-0.80)$ & $<0.0001$ \\
\hline
\end{tabular}

Abbreviations: $\mathrm{Cl}$, confidence interval; IBD, inflammatory bowel disease; $\mathrm{OR}$, odds ratio.

${ }^{a}$ Adjusted prevalence (95\% Cl): IBD 6.39\% (range: 6.31-6.47\%) versus no IBD 3.44\% (range: $3.43-3.44 \%$ ).

more common in IBD than non-IBD thrombosis patients, consistent with previous studies. ${ }^{10,16-21}$ Malnutrition was likely associated with other risks for VTE, including requirement for port/central venous catheter, hospitalization, and/or surgery. ${ }^{19}$ Further, women with IBD, similar to those without IBD, were significantly more likely than men to develop thrombosis, as previously reported, consistent with thrombosis risk in pregnancy and in the postpartum period. ${ }^{22,23}$

Asian ethnicity was found to be protective, consistent with general risk of thromboembolism in this group. ${ }^{24,25} \mathrm{By}$ contrast, typical risk factors for VTE, including hypertension, hyperlipidemia, cardiac disease, diabetes, renal failure, and obesity, were significantly lower in IBD with thrombosis, likely, in part, related to their lower age.

Whether any risk groups for thrombosis among those with IBD would benefit from anticoagulation prophylaxis remains unknown. Thromboprophylaxis is typically avoided in patients with IBD due to concerns regarding GI tract bleeding. ${ }^{26}$ Despite this, anticoagulation prophylaxis has been shown to be safe in IBD patients, with no greater bleeding than in non-IBD patients. ${ }^{18,26,27}$ Further, it has been recommended that anticoagulation should be extended beyond acute thrombosis and/or hospitalization, ${ }^{18,28,29}$ especially in those with frequent flares and chronic steroid use. ${ }^{17,18,30,31}$ Our findings indicate that thrombosis risk exists in other subsets of IBD patients, that is, those using ports and undergoing general and 
abdominal surgery. In fact, it has been shown that IBD patients receiving thromboprophylaxis within 24 hours of admission are half as likely to develop VTE. ${ }^{18}$ However, more research is needed to identify those who might benefit from long-term anticoagulation.

The observation that thrombosis site differs between those with IBD and those without IBD is of interest. Specifically, those with IBD were nearly twice as likely to have mesenteric or portal vein thrombosis, but significantly less likely to have pulmonary embolism, deep venous thrombosis, cerebral arterial embolism, or cerebral arterial thrombosis than in those without IBD. This suggests the possibility that the pathophysiology of IBD may involve prothrombotic signaling or pathways that promote local thrombosis in the GI tract. Further mechanistic studies of IBD thrombosis might lead to a better understanding of thrombosis, in general, and potentially identify new targets for thrombosis prevention.

Why mortality was significantly lower in individuals with IBD, compared with those without IBD is unknown. It is possible, but not proven that lower IBD mortality could be related to the lower prevalence of African American ethnicity, a group with poorer access to care and poorer health outcomes; or the lower IBD mortality could be related to a lower prevalence of malignancy and associated shortened survival. However, these are not proven, and more research is needed to determine the causes for reduced mortality in IBD.

\section{Limitations}

There are several limitations to this study. First, as the NIS is an inpatient database, there is potential bias toward a sicker population, as healthier patients who did not require admission are not included, introducing selection bias. Second, the NIS represents only $20 \%$ of the total inpatient population, so discharge-level-weights were used to determine a representative sample. Third, this sample is dependent on discharge diagnoses codes, which are limited by coding accuracy and potential misclassification bias, and further prevent tracking of the individual patient as the data are of discharge level rather than patient level. For the same reason, it is not possible to determine whether thrombosis at the patient level was provoked, symptomatic, or whether thromboprophylaxis was given. However, previous studies examining hospital discharge data have indicated sufficient accuracy for use in research studies. $^{32,33}$ Fourth, as this was a retrospective study, it is subject to bias and cannot be used to determine causality. Fifth, the NIS database does not contain laboratory values or drug treatment information, and thus it cannot be used to assess the relation between thrombosis risk and severity of IBD, duration of steroids or port use, or use of thromboprophylaxis, nor is it possible to adjust results for IBD duration or disease activity. A significant strength of the NIS is its size, this sample offers a large patient pool to assess an uncommon disease like IBD.

\section{Conclusion}

In conclusion, this study confirms that venous thrombosis is significantly increased among IBD patients. Recognition of those at high risk may help to identify potential patients for thromboprophylaxis safety and efficacy studies. Future trials will be critical to develop evidence for the optimal management of these patients.

\section{Authors' Contributions}

J.B.C. and M.V.R. contributed to the study design, data acquisition, interpretation of the data and writing of the manuscript. D.M.C. contributed to data acquisition, performance of the data analysis, and critical review of the manuscript. J.G.Y. contributed to the study design, data acquisition, performance of the data analysis, and critical review of the manuscript. The dataset and analysis of the selected years from the NIS can be obtained by contacting the corresponding author.

\section{Funding}

This study was supported, in part, by Health Resources \& Services Administration (HRSA) Federal Hemophilia Treatment centers Grant, 4500 Fishers Lane, Rockville, MD 20857 (Grant H30MC24050-04-00); National Institutes of Heart, Lung, Blood Institute (NHLBI), Building 31, 31 Center Drive, Bethesda, MD 20892 (NHLBI 2T35HL074708-13); and Pennsylvania Department of Health (DOH), Harrisburg, PA 17108, State Support of Hemophilia Center of Western PA (SAP no.: 41000058531).

Conflict of Interest

None declared.

\section{References}

1 Loftus EV Jr. Clinical epidemiology of inflammatory bowel disease: Incidence, prevalence, and environmental influences. Gastroenterology 2004;126(06):1504-1517

2 Kappelman MD, Rifas-Shiman SL, Kleinman K, et al. The prevalence and geographic distribution of Crohn's disease and ulcerative colitis in the United States. Clin Gastroenterol Hepatol 2007;5 (12):1424-1429

3 Molodecky NA, Soon IS, Rabi DM, et al. Increasing incidence and prevalence of the inflammatory bowel diseases with time, based on systematic review. Gastroenterology 2012;142(01):46-54. e42, quiz e30

4 Strober W, Fuss IJ. Proinflammatory cytokines in the pathogenesis of inflammatory bowel diseases. Gastroenterology 2011;140(06): 1756-1767

5 Neurath MF. Cytokines in inflammatory bowel disease. Nat Rev Immunol 2014;14(05):329-342

6 Esmon CT. Inflammation and thrombosis. J Thromb Haemost 2003;1(07):1343-1348

7 Senchenkova E, Seifert H, Granger DN. Hypercoagulability and platelet abnormalities in inflammatory bowel disease. Semin Thromb Hemost 2015;41(06):582-589

8 Alkim H, Ayaz S, Alkim C, Ulker A, Sahin B. Continuous active state of coagulation system in patients with nonthrombotic inflammatory bowel disease. Clin Appl Thromb Hemost 2011;17(06):600-604

9 Bernstein CN, Blanchard JF, Houston DS, Wajda A. The incidence of deep venous thrombosis and pulmonary embolism among patients with inflammatory bowel disease: a population-based cohort study. Thromb Haemost 2001;85(03):430-434

10 Grainge MJ, West J, Card TR. Venous thromboembolism during active disease and remission in inflammatory bowel disease: a cohort study. Lancet 2010;375(9715):657-663 
11 Kappelman MD, Horvath-Puho E, Sandler RS, et al. Thromboembolic risk among Danish children and adults with inflammatory bowel diseases: a population-based nationwide study. Gut 2011; 60(07):937-943

12 Nguyen GC, Bernstein CN, Bitton A, et al. Consensus statements on the risk, prevention, and treatment of venous thromboembolism in inflammatory bowel disease: Canadian Association of Gastroenterology. Gastroenterology 2014;146(03):835-848.e6

13 Tinsley A, Naymagon S, Trindade AJ, Sachar DB, Sands BE, Ullman TA. A survey of current practice of venous thromboembolism prophylaxis in hospitalized inflammatory bowel disease patients in the United States. J Clin Gastroenterol 2013;47(01):e1-e6

14 Sam JJ, Bernstein CN, Razik R, Thanabalan R, Nguyen GC. Physicians' perceptions of risks and practices in venous thromboembolism prophylaxis in inflammatory bowel disease. Dig Dis Sci 2013;58(01):46-52

15 Databases HCUP; Healthcare Cost and Utilization Project (HCUP). Overview of the National (Nationwide) Inpatient Sample (NIS). Available at: www.hcup-us.ahrq.gov/nisoverview.jsp. Accessed April 20, 3030

16 Kaplan GG, Lim A, Seow CH, et al. Colectomy is a risk factor for venous thromboembolism in ulcerative colitis. World J Gastroenterol 2015;21(04):1251-1260

17 Higgins PDR, Skup M, Mulani PM, Lin J, Chao J. Increased risk of venous thromboembolic events with corticosteroid vs biologic therapy for inflammatory bowel disease. Clin Gastroenterol Hepatol 2015;13(02):316-321

18 Kaddourah O, Numan L, Jeepalyam S, Abughanimeh O, Ghanimeh MA, Abuamr K. Venous thromboembolism prophylaxis in inflammatory bowel disease flare-ups. Ann Gastroenterol 2019;32(06):578-583

19 Egberg MD, Galanko JA, Barnes EL, Kappelman MD. Thrombotic and infectious risks of parenteral nutrition in hospitalized pediatric inflammatory bowel disease. Inflamm Bowel Dis 2019;25 (03):601-609

20 Rooden CJ, Tesselaar ME, Osanto S, Rosendaal FR, Huisman MV. Deep vein thrombosis associated with central venous catheters - a review. J Thromb Haemost 2005;3(11):2409-2419

21 Najjar PA, Madenci AL, Zogg CK, et al. Implementation of a comprehensive post-discharge venous thromboembolism prophylaxis program for abdominal and pelvic surgery patients. J Am Coll Surg 2016;223(06):804-813
22 Morgan K, Boktor M, Ford C, et al. Venous thromboembolism in IBD: Increased risk for women in CD? Pathophysiology 2019;26 (02):163-168

23 Kim YH, Pfaller B, Marson A, Yim HW, Huang V, Ito S. The risk of venous thromboembolism in women with inflammatory bowel disease during pregnancy and the postpartum period: A systematic review and meta-analysis. Medicine (Baltimore) 2019;98 (38):e17309

24 Zakai NA, McClure LA. Racial differences in venous thromboembolism. J Thromb Haemost 2011;9(10):1877-1882

25 Klatsky AL, Armstrong MA, Poggi J. Risk of pulmonary embolism and/or deep venous thrombosis in Asian-Americans. Am J Cardiol 2000;85(11):1334-1337

26 Faye AS, Hung KW, Cheng K, et al. Minor hematochezia decreases use of venous thromboembolism prophylaxis in patients with inflammatory bowel disease. Inflamm Bowel Dis 2019 (e-pub ahead of print); Doi: 10.1093/ibd

27 Lobo JL, Garcia-Fuertes JA, Trujillo-Santos J, et al; RIETE Investigators. Anticoagulant therapy for venous thromboembolism in patients with inflammatory bowel disease. Eur J Gastroenterol Hepatol 2018;30(05):526-530

28 Ra G, Thanabalan R, Ratneswaran S, Nguyen GC. Predictors and safety of venous thromboembolism prophylaxis among hospitalized inflammatory bowel disease patients. J Crohn's Colitis 2013; 7(10):e479-e485

29 Nguyen GC, Murthy SK, Bressler B, et al; CINERGI group. Quality of care and outcomes among hospitalized inflammatory bowel disease patients: a multicenter retrospective study. Inflamm Bowel Dis 2017;23(05):695-701

30 Nguyen GC, Bernstein CN. Duration of anticoagulation for the management of venous thromboembolism in inflammatory bowel disease: a decision analysis. Am J Gastroenterol 2013;108(09): 1486-1495

31 Alkim H, Koksal AR, Boga S, Sen I, Alkim C. Etiopathogenesis, prevention, and treatment of thromboembolism in inflammatory disease. Clin Appl Thromb Hemost 2017;23(06):501-510

32 Sarrazin MS, Rosenthal GE. Finding pure and simple truths with administrative data. JAMA 2012;307(13):1433-1435

33 Haut ER, Pronovost PJ, Schneider EB. Limitations of administrative databases. JAMA 2012;307(24):2589-2590, author reply 2589-2590 